\title{
Role of OCT- A in glaucoma
}

\author{
Priyanka R, Karthikeyan M, Dewang A*, Shorya VA and Ramanjit S \\ Dr RP Centre for Ophthalmic Sciences, AIIMS, India
}

*Corresponding author: Dewang Angmo, Assistant Professor, Dr RP Centre for Ophthalmic Sciences, AIIMS, Third floor Dr. R. P. Centre for Ophthalmic Sciences All India Institute of Medical Sciences Ansari Nagar, New Delhi, India-110029, India, Tel: 9810896270; Email: dewang45@gmail.com

\section{Mini Review}

Volume 3 Issue 6

Received Date: October 12, 2018

Published Date: December 21, 2018

DOI: 10.23880 /oajo- 16000170
Abbreviations: OCT: Optical Coherence Tomography; OCTA: Optical Coherence Tomography Angiography; FA: Fluorescein Angiography; ICGA: Indocyanine Green Angiography; PAFD: Peripapillaryangio Flow Density; RNFL: Retinal Nerve Fiber Layer; IOP: Intra Ocular Pressure.

\section{Introduction}

Optical coherence tomography (OCT) is a non-contact, non-invasive imaging system that uses low coherence interferometry [1]. High-resolutioncross-sectional images are produced by analysis of interference between the reflected reference waves and those reflected by the tissues. With the improvement in sensitivity, acquisition speed and resolution, it has been widely used for micro structural analysis of the ocular structures [1].

The conventional methods of assessing the posterior segment circulation include fluorescein angiography and Indocyanine green angiography. Fluorescein angiography is used for visualizing retinal vessels and Indocyanine Green angiography is used for visualizing the choroidal vasculature. These methods are time-consuming and also have been associated with various systemic adverse effects [1].

Optical coherence tomography angiography (OCTA) is a new non-invasive en face imaging technique used to image retinal vasculature [2]. It creates a contrast between the blood vessels and the surrounding retinal tissue by assessing the changes in the signals by the moving RBCs. These results in an intrinsic contrast called speckled variance/ decorrelation. It creates angiogram by separating the moving scattered rays of light from the static background reflected light [1]. The technology currently used is called as Split Spectrum Amplitude- de correlation angiography. Instead of comparing adjacent A- scans, OCTA compares the de correlation signal between sequential OCT b-scans to construct a map of blood flow. It reduced the noise and improves the resolution of images ${ }^{1}$.

OCTA has been widely used in retinal pathologies like in diabetic retinopathy and vascular occlusions to study the retinal capillary drop out, to study the foveal avascular zone and to study new vessel formation. It was given more knowledge about choroidal vasculature and the pachychoroid spectrum of diseases. It has been used in choroideremia, macular telangiectasia, dry ARMD and also in the identification and characterization of choroidal neovascular membranes $[3,4]$. It has been used in the study of optic nerve head perfusion in glaucoma and in multiple sclerosis $[1,5]$.

OCTA is a better alternative for patients in whom fluorescein or indocyanine green dyes are contraindicated due to allergy, pregnancy or kidney disease and have the advantage of giving a three-dimensional view as opposed to conventional angiogram which is a two-dimensional image [6]. In fluorescein angiography (FA) and indocyanine green angiography (ICGA) leakage of dye can obscure retinal pathology. This difficulty is overcome in OCTA as no dye is injected. OCTA has better resolution and provides exact location and size measurements of the pathology. OCTA is used for visualizing both retinal and choroidal microvasculature [7].

OCTA has its own set of disadvantages. There can be many artifacts due to the intrinsic property of the eye, image acquisition, eye motion and the analysis of the image and display. There can be "Projection artifacts" in which structures in the superficial layer will also be seen in the deeper layers. Eye movement is another major cause of image distortion and artifacts. Eye movement causes a change in the successive B scans and hence these 
artifacts appear as white lines. There can also be an error in the segmentation algorithm and hence the structures seen might not correlate anatomically. The other drawback seen with OCTA is that it cannot detect a leak from a vessel [8]. OCTA is also less likely to pick up lesions which have low flow velocities like microaneurysms or fibrotic choroidal neovascularization.

\section{Role of OCTA in glaucoma}

Ocular blood flow, particularly optic disc perfusion is thought to play an important role in the development and progression of glaucoma $[9,10]$. OCTA has been used to study the optic disc perfusion [1]. The reduced peripapillary perfusion in glaucomatous eyes can be visualized as focal defects. It can be quantified as peripapillary flow index and peripapillary vessel density [11]. The mean decorrelation value on en face retinal angiogram is defined as peripapillary flow index. It is the measure of the area of large vessels and area velocity of retinal microvasculature. Flow index calculated is highly sensitive and specific to differentiate glaucomatous eyes from normal eyes [12]. The percentage of area occupied by the large vessels and microvasculature in the peripapillaryregion is defined as peripapillary vessel density.

Wang et al. used OCTA to explore the optic disc perfusion (flow index and vessel density) in open-angle glaucoma patients and its correlation with disease severity [13]. Reduced disc flow index and vessel density were demonstrated in glaucoma patients. This reduction was strongly related to ganglion cell complex thickness. They concluded that measurement of disc perfusion by OCTA may be important for monitoring glaucoma.

Gabor Hollo analyzed the peripapillary angio flow density (PAFD) in superotemporal, inferotemporal, temporal peripapillary sectors and its relationship with retinal nerve fiber layer (RNFL) damage [14]. He suggested that decreased peripapillary perfusion can be identified by PAFD measurements and it occurs prior to the development of clinically significant RNFL damage or visual field loss. Thus calculating sector PAFD by OCTA may help in detecting pre-perimetric glaucoma earlier than the time at which RNFL OCT could detect.

Several studies reported the reduction of vessel density in sectors with RNFL defect in both primary open angle glaucoma and primary angle closure glaucoma [1517]. The reduction in vessel density was greater in the peripapillary region compared to the parafoveal region.
Jung et al. demonstrated a significant increase in peripapillary vessel density after trabeculectomy in glaucomatous eyes [18]. This increase in vessel density is attributed to the postoperative reduction in intra ocular pressure (IOP) which improves the ocular perfusion. Thus OCTA can aid in monitoring the progression and response to treatment in glaucomatous eyes.

Yarmohammadi, et al. evaluated the relationship between the retinal vessel density and visual field loss in glaucoma [19]. There was a significant association between the decreased vessel density and severity of visual field loss. This association was found to be stronger compared to measurements like RNFL thickness and rim area. Vessel density measured by OCTA can serve as an adjuvant (better than RNFL thickness) to the visual field in glaucoma patients.

Akagi, et al. evaluated the optic disc microvasculature dropout (Mvd-D) using OCTA in glaucomatous eyes [20]. $\mathrm{He}$ used visualization of anterior lamina cribriosa to eliminate pseudo Mvd-D. Patients with Mvd-D also have lamina cribriosa defects and microvascular dropouts in the parapapillary region. There was a significant association of Mvd-D with larger cup-disk ratio, worse visual field mean deviation.

\section{Our Experience}

In our center, we have evaluated the perfusion density and the vessel density in 24 patients of glaucoma using ZEISS Angioplex OCT angiography machine. We had a total of 14 patients with primary open angle glaucoma, 5 with primary angle closure glaucoma and 5 with combined mechanism glaucoma. The measurements were done in the peripapillary area using the inbuilt algorithm. The peripapillary area was divided into superior, inferior, nasal and temporal areas and measurements were taken in each of the 4 quadrants. The mean vessel density in the superior quadrant was found to be $16.93+/-1.56$ and the perfusion density was $0.429+/-0.0454$. In the inferior quadrant, the vessel density was $15.98+/-1.74$ and the perfusion pressure was $0.405+/-0.049$. In the temporal quadrant, the vessel density was $17.23+/-2.6$ and perfusion density was $0.42+/-0.065$. In the nasal quadrant, the vessel density was $16.02+/-2.35$ and perfusion density was $0.402+/-0.064$. On the whole, the vessel density was $16.7+/-0.99$. Further analysis is still needed to see the changes when compared with controls and to see if there are any changes between the different kinds of glaucomas. 


\section{Conclusion}

OCTA is a newly introduced non-invasive imaging modality to visualize retinal vasculature. In glaucoma patients, it detects optic disc perfusion quantitatively using flow index and vessel density and can help in early diagnosis, monitoring progression and to observe the effectiveness of treatment. Further long-term studies need to done to establish its role in glaucoma patients.

\section{References}

1. Gao SS, Jia Y, Zhang M, Su JP, Liu G, et al. (2016) Optical coherence tomography angiography. IOVS 57(9): ОСТ27-ОСТ36.

2. Jia Y, Tan O, Tokayer J, Potsaid B, Wang Y, et al. (2012) Split-spectrum amplitude-decorrelation angiography with optical coherence tomography. Optics Express 20(4): 4710-4725.

3. Jia Y, Bailey ST, Wilson DJ, Tan O, Klein ML, et al. (2014) Quantitative optical coherence tomography angiography of choroidal neovascularization in agerelated macular degeneration. Ophthalmology 121(7): 1435-1444.

4. de Carlo TE, Bonini Filho MA, Chin AT, Adhi M, Ferrara D, et al. (2015) Spectral-domain optical coherence tomography angiography of choroidal neovascularization. Ophthalmology 122(6): 12281238.

5. Jia Y, Morrison JC, Tokayer J, Tan O, Lombardi L, et al. (2012) Quantitative OCT angiography of optic nerve head blood flow. Biomed Opt Express 3(12): 31273137.

6. Talisa E de Carlo, Andre Romano, Nadia K Waheed, Duker JS (2015) A review of optical coherence tomography angiography (OCTA). Int J Retina Vitr 1(1): 5 .

7. Campbell JP, Zhang M, Hwang TS, Bailey ST, Wilson DJ, et al. (2017) Detailed Vascular Anatomy of the Human Retina by Projection-Resolved Optical Coherence Tomography Angiography. Sci Rep 7: 42201.

8. Spaide RF, Fujimoto JG, Waheed NK (2015) Image Artifacts in Optical Coherence Tomography Angiography. Retina 35(11): 2163-2180.
9. Kornzweig AL, Eliasoph I, Feldstein M (1968) Selective atrophy of the radial peripapillary capillaries in chronic glaucoma. Arch Ophthalmol 80(6): 696-702.

10. Flammer J (1994) The vascular concept of glaucoma. Surv Ophthalmol 38: S3-S6.

11. Liu L, Jia Y, Takusagawa HL, Pechauer AD, Edmunds B, et al. (2015) Optical coherence tomography angiography of the peripapillary retina in glaucoma. JAMA Ophthalmol 133(9): 1045-1052.

12. Jia $Y$, Wei E, Wang $X$, Zhang $X$, Morrison JC, et al. (2014) Optical coherence tomography angiography of optic disc perfusion in glaucoma. Ophthalmology 121(7): 1322-1332.

13. Wang $X$, Jiang $C$, Ko T, Kong $X$, Yu X, et al. (2015) Correlation between optic disc perfusion and glaucomatous severity in patients with open-angle glaucoma: an optical coherence tomography angiography study. Graefes Arch Clin Exp Ophthalmol 253(9): 1557-1564.

14. Holló G (2016) Vessel Density Calculated from OCT Angiography in 3 Peripapillary Sectors in Normal, Ocular Hypertensive, and Glaucoma Eyes. Eur J Ophthalmol 26(3): e42-45.

15. Lee EJ, Lee KM, Lee SH, Kim TW (2016) OCT Angiography of the Peripapillary Retina in Primary Open-Angle Glaucoma. Invest Ophthalmol Vis Sci 57(14): 6265-6270.

16. Zhu L, Zong Y, Yu J, Jiang C, He Y, et al (2018) Reduced Retinal Vessel Density in Primary Angle Closure Glaucoma: A Quantitative Study Using Optical Coherence Tomography Angiography. J Glaucoma 27(4): 322-327.

17. Suh MH, Zangwill LM, Manalastas PIC, Belghith A, Yarmohammadi A, et al. (2016) Optical Coherence Tomography Angiography Vessel Density in Glaucomatous Eyes with Focal Lamina Cribrosa Defects. Ophthalmology 123(11): 2309-2317.

18. In JH, Lee SY, Cho SH, Hong YJ (2018) Peripapillary Vessel Density Reversal after Trabeculectomy in Glaucoma. Journal of Ophthalmology pp: 7.

19. Yarmohammadi A, Zangwill LM, Diniz-Filho A, Suh MH, Yousefi S, et al. (2016) Relationship between Optical Coherence Tomography Angiography Vessel 


\section{Open Access Journal of Ophthalmology}

Density and Severity of Visual Field Loss in Glaucoma. Ophthalmology 123(12): 2498-508.

20. Akagi T, Zangwill LM, Shoji T, Suh MH, Saunders LJ, et al. (2018) Optic disc microvasculature dropout in primary open-angle glaucoma measured with optical coherence tomography angiography. PloS One 13(8): e0201729.

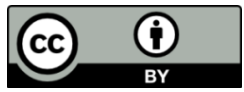

